\title{
The Riemann Hypothesis Is not Equality
}

\author{
Wu Hefa* \\ Freedom Mathematical Researchers, Handong Heze Shanxian, China. \\ * Corresponding author. Tel.: 15253094043; email: 150896091@qq.com \\ Manuscript submitted April 12, 2016, accepted November 30, 2016. \\ doi: 10.17706/ijapm.2017.7.2.134-140
}

\begin{abstract}
The Riemann hypothesis is the most important in today's international maths, expect to solve the most famous incomplete mathematics problem, it is incomplete in the classical mathematical analysis form the product of paradox. As long as the classical mathematical analysis system does not get rid of their incomplete defects, Riemann hypothesis cannot completely solve, even the most basic also failed to set up or not. But what is of the Riemann hypothesis? Why is it important? Still not had a relatively complete system of certificate for introduction, explanation and elaboration, only the reference of existing irrelevant answer of so-called "mathematics specialized books, documents or ramble", rather than the law of trust in science. So even all mathematicians not to mention ordinary people will only know "the Riemann hypothesis" the question. Riemann hypothesis is not completely equation solved this difficult problem.
\end{abstract}

Key words: Euler product, the reciprocal law of natural numbers, The Riemann hypothesis is not equal.

\section{Introduction}

\subsection{Euler's Proof of Euler's Constnta}

Euler in his paper some review of the Infinite Series (Various Observations about Infinite Series) in the product of Euler formula "to" prove Riemann zeta function, and published in 1737by the academy of sciences. Riemann zeta function can be written in the form of Euler's product [1].

$$
\varsigma_{(\mathrm{s})}=\sum \frac{1}{n^{s}}=1+\frac{1}{2^{s}}+\frac{1}{3^{s}}+\frac{1}{4^{s}}+\ldots \ldots,
$$

and the left is equal to the Riemann zeta function, the right product is extended to all prime number $\mathrm{p}$

$$
\prod_{\mathrm{p} \text { prime }} \frac{1}{1-\mathrm{p}^{-s}}=\frac{1}{1-2^{-s}} \cdot \frac{1}{1-3^{-s}} \cdot \frac{1}{1-5^{-s}} \cdot \frac{1}{1-7^{-s}} \cdots \frac{1}{1-p^{-s}} \ldots
$$

Prove process only to form simple and omit the infinite, it is the incomplete method that the Euler once used. From (2) less (1), all the even items: type, we get rid of [2]

$$
\zeta(\mathrm{s})=1+\frac{1}{2^{s}}+\frac{1}{3^{s}}+\frac{1}{4^{s}}+\ldots, \quad \frac{1}{2^{s}} \zeta(s)=\frac{1}{2^{s}}+\frac{1}{4_{s}}+\frac{1}{6^{s}}+\frac{1}{8^{s}}+\ldots
$$




$$
\begin{aligned}
& \frac{1}{3^{s}}\left(1-\frac{1}{2^{s}}\right) \varsigma_{(s)}=\frac{1}{3^{s}}+\frac{1}{9^{s}}+\frac{1}{15^{s}}+\frac{1}{21^{s}}+\frac{1}{27^{s}}+\ldots \\
& \left(1-\frac{1}{3^{s}}\right)\left(1-\frac{1}{2^{s}}\right) \zeta(s)=1+\frac{1}{5^{s}}+\frac{1}{7^{s}}+\frac{1}{11^{s}}+\frac{1}{13^{s}}+\frac{1}{17^{s}}+\ldots
\end{aligned}
$$

The cut all multiple item 2 and 3. Multiples items can be visible to the right, to repeat the above steps are sieve to available:

$$
\ldots\left(1-\frac{1}{11^{s}}\right)\left(1-\frac{1}{7^{s}}\right)\left(1-\frac{1}{5^{s}}\right)\left(1-\frac{1}{3^{s}}\right)\left(1-\frac{1}{2^{s}}\right) \zeta(s)=1
$$

At last, Euler got

$$
\varsigma_{(S)}=\prod_{P, \text { Prime }} \frac{1}{1-p^{-s}}[3]
$$

To prove more closely, we need to notice when $\mathrm{R}(\mathrm{s})>1$, sieved the right item to 1 , and follow the convergence of dirichlet series. Is derived from the above all can zeta the interesting but the results of the paradox.

$$
\ldots\left(1-\frac{1}{11}\right)\left(1-\frac{1}{7}\right)\left(1-\frac{1}{5}\right)\left(1-\frac{1}{3}\right)\left(1-\frac{1}{2}\right) \varsigma_{(1)}=1
$$

It can be written as ......(10/11)(6/7)(4/5)(2/3)(1/2)ל (1)=1,

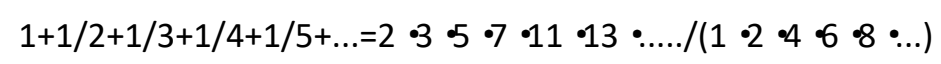

Euler proved to be incorrect, derived [4] $1+\frac{1}{2}+\frac{1}{3}+\frac{1}{4}+\ldots \ldots+\frac{1}{\mathrm{n}}=\ln (n+1)+r$

\section{Euler's Proof Is not Complete, the Following to Correct}

\subsection{The Euler's Constant r (Euler - Maxieluoni Constant) Are Irrational Numbers [5].}

The source of the Euler's constant $r$ : Euler's constant $r$ is objective existence, it is indisputable fact that the number of frequency - Euler's constant $r$ is already fully respect Euler found that with the inevitable number frequency conditions, the frequency from several science, so it is the series of classical mathematics to several scientific frequency of a leap, number and frequency law make us believe that the objective of this explanation is reasonable. In 1734 , Euler using Newton's achievements, won the first harmonic finite terms the value of the sum of the series. $1+1 / 2+1 / 3+\cdots+1 / n=\ln (n+1)+r$, he process is as follows: By $\ln (1+x)=x-x^{2} / 2+x^{3} / 3-x^{4} / 4+\cdots, \quad(-1<x \leqslant 1), x=1,2,3,4, \ldots, 1 / n$, $\ln (1+1 / n)=\ln [(n+1) / n]=1 / n-1 / 2 \cdot 1 / n^{2}+1 / 3 \cdot 1 / n^{3}-1 / 4 \cdot 1 / n^{4}+\cdots ;$

The above all kinds of additive, and tidy:

$$
\begin{array}{r}
1+1 / 2+1 / 3+1 / 4+\cdots+1 / n=\ln (1+n)+r, r=1 / 2\left(1 / 2^{2}+1 / 3^{2}+1 / 4^{2} \cdots+1 / n^{2}\right)-1 / 3\left(1 / 2^{3}+1 / 3^{3}+1 / 4^{3}+\cdots\right) \\
+1 / 4\left(1 / 2^{4} 4+1 / 3^{4} 4+1 / 4^{4} 4+\cdots\right)-1 / 5\left(1 / 2^{5} 5+1 / 3^{5} 5+1 / 4^{5}+\cdots\right)+\cdots=1 / 2 \sum 1 / n^{2}-1 / 3 \sum 1 / n^{3}+1 / 4 \sum 1 / n^{4}-1 / 5 \sum
\end{array}
$$




$$
1 / \mathrm{n}^{5}+\cdots+\left[(-1)^{\mathrm{n}-1}\right] / \mathrm{n} \sum 1 / \mathrm{n}^{\mathrm{n}} \cdot \quad(\mathrm{n}=2, \infty)
$$

This is a youth mathematician Euler make great contributions to harmonic progression and analysis, it is the modern theory of modern classic series is the important measure to avoid.

\subsection{The Reciprocal Law of the Reciprocal of Natural Numbers}

$$
\begin{gathered}
1 / \mathrm{n}=1 /(1+\mathrm{n})+1 /(1+\mathrm{n}) 2+1 /(1+\mathrm{n}) 3+\ldots+1 /(1+\mathrm{n}) \mathrm{n}-1+1 / \mathrm{n} \cdot 1 /(1+\mathrm{n}) \mathrm{n} . \\
\mathrm{n}=1, \quad 1=1 / 2+1 / 2^{2}+1 / 2^{3}+1 / 2^{4}+\cdots=1 / 2\left(1-1 / 2^{\mathrm{n}}\right) /(1-1 / 2)+1 \cdot(1 / 2)^{\mathrm{n}}=1 ; \\
\mathrm{n}=2,1 / 2=1 / 3+1 / 3^{2}+1 / 3^{3}+1 / 3^{4}+\cdots=1 / 3\left(1-1 / 3^{\mathrm{n}}\right) /(1-1 / 3)+1 / 2 \cdot\left(1 / 3^{\mathrm{n}}\right) \\
\mathrm{n}=3,1 / 3=1 / 4+1 / 4^{2}+1 / 4^{3}+1 / 4^{4}+\cdots=1 / 4\left(1-1 / 4^{\mathrm{n}}\right) /(1-1 / 4)+1 / 3 \cdot\left(1 / 4^{\mathrm{n}}\right) \\
\cdots \quad \cdots \quad \cdots \quad \cdots
\end{gathered}
$$

The law of the number and frequency of scientific significance is that it's identity, is not imprecise approximation theory of classical mathematics, regardless of any natural number $\mathrm{n}$ is set up.The above all together, the left $=1+1 / 2+1 / 3+1 / 4+\cdots+1 / \mathrm{n}$

The right $=[1 / 2+1 / 3+1 / 4+\cdots+1 /(\mathrm{n}+1)]+\left[1 / 2^{2}+1 / 3_{2}+1 / 4^{2}+\cdots+1 /(\mathrm{n}+1)^{2}\right]+\left[1 / 2^{3}+1 / 3^{3}+1 / 4^{3}+\cdots\right.$ $\left.+1 /(n+1)^{3}\right]+\left[1 / 2^{4}+1 / 3^{4}+1 / 4^{4}+\cdots+1 /(n+1)^{4}\right]+\cdots$ On the left on the right side of the equal,

So

$$
1-1 /(n+1)=\left[1 / 2^{2}+1 / 3^{2}+1 / 4^{2}+\cdots\right]+\left[1 / 2^{3}+1 / 3^{3}+1 / 4^{3}+\cdots\right]+\cdots
$$

$\therefore \mathrm{n} \rightarrow \infty \quad$ so $1=\left[1 / 2^{2}+1 / 3^{2}+1 / 4^{2}+\cdots\right]+\left[1 / 2^{3}+1 / 3^{3}+1 / 4^{3}+\cdots\right]+\left[1 / 2^{4}+1 / 3^{4}+1 / 4^{4} \quad \cdots \quad\right]+\cdots$ ，

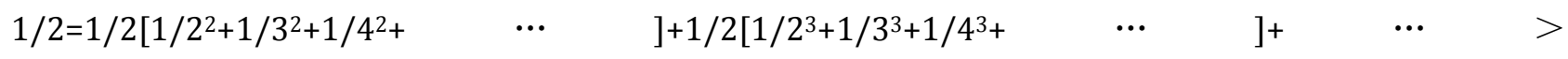
$1 / 2\left[1 / 2^{2}+1 / 3^{2}+1 / 4^{2}+\cdots\right]-1 / 3\left[1 / 2^{3}+1 / 3^{3}+\cdots\right]+1 / 4\left[1 / 2^{4}+1 / 3^{4}+\cdots\right]-\cdots=r>0$,

Then $1 / 2>r>0$. This is the number of frequency - Euler's constant $r$ preliminary conclusions. Prove that $0<r<1 / 3$, but at the same time prove that $r$ is irrational. According to the results of Euler and others: $0<r$ $<1 / 3, \quad \mathrm{n} \rightarrow \infty$, There is [6]

$\zeta_{(2)}=1+1 / 2^{2}+1 / 3^{2}+1 / 4^{2}+\cdots=\left(\pi^{2}\right) / 6$;

$\zeta_{(3)}=1+1 / 2^{3}+1 / 3^{3}+1 / 4^{3}+\cdots=1.202056903 \cdots$ Apéry's constant

$\left.\zeta_{(4)}=1+1 / 2^{4}+1 / 3^{4}+1 / 4^{4}+\cdots=(\pi){ }^{4}\right) / 90=1.0823 \cdots$;

$\zeta_{(5)}=1+1 / 2^{5}+1 / 3^{5}+1 / 4^{5}+\cdots=1.03692775 \cdots$,

$\zeta_{(6)}=1+1 / 26+1 / 36+1 / 46+\cdots=\left(\right.$ п $\left.{ }^{6}\right) / 945=1.002476 \cdots$;

$\zeta_{(7)}=1.00083492773879228 \ldots . .$.

$\zeta_{(8)}=1+1 / 2+1 / 3+1 / 4+\cdots=\left(\pi^{8}\right) / 9450=1.0039827 \cdots ; \cdots \cdots$

Clearly $\zeta_{(2)}>\zeta_{(3)}>\zeta_{(4)}>\zeta_{(5)}>\zeta_{(6)}>\zeta{ }_{(7)}>\zeta{ }_{(8)}>\cdots \cdots$.

Let $\mathrm{R}=1 / 2 \zeta \quad(2)^{-1 / 3 \zeta} \quad{ }_{(3)}+1 / 4 \zeta \quad(4)^{-1 / 5 \zeta} \quad{ }_{(5)}{ }^{+\cdots+(-1)^{(n-1)}(\zeta \quad n) / n}$

$$
=1 / 2\left(1+1 / 2^{2}+1 / 3^{2}+1 / 4^{2}+\cdots\right)-1 / 3\left(1+1 / 2^{3}+1 / 3^{3}+1 / 4^{3}+\cdots\right)+1 / 4\left(1 / 2^{4}+1 / 3^{4}+1 / 4^{4}+\cdots\right)-\cdots
$$




$$
\begin{gathered}
+(-1)^{\mathrm{n}-1}\left(1+1 / 2^{\mathrm{n}}+1 / 3^{\mathrm{n}}+1 / 4^{\mathrm{n}}+\cdots\right) / \mathrm{n}=(1 / 2-1 / 3+1 / 4-1 / 5+\cdots)+\left[1 / 2 \sum 1 / \mathrm{n}^{2}-1 / 3 \sum 1 / \mathrm{n}^{3}+1 / 4 \sum 1 / \mathrm{n}^{4}-1 / 5 \sum\right. \\
\left.1 / \mathrm{n}^{5}+\cdots\right], \quad=(1-\ln 2)+\mathrm{r},(\mathrm{n}=2, \infty)
\end{gathered}
$$

Because $\mathrm{R}$ is alternating series number of diminishing the deformation of culvert, according to the alternating series on the folding method, always have $R<\frac{1}{2} \varsigma_{(2)}-\frac{1}{3} \varsigma_{(3)}+\frac{1}{4} \varsigma_{(4)}-\frac{1}{5} \varsigma_{(5)}+\frac{1}{6} \varsigma_{(6)}-\frac{1}{7} \varsigma_{(7)}+\frac{1}{8} \varsigma_{(8)}<\frac{\varsigma_{(2)}}{2}, \quad \frac{\varsigma_{(7)}}{7}$ using zeta $\frac{\varsigma_{(8)}}{8}$ instead it still exists $R<\frac{S_{(2)}}{2}-\frac{S_{(3)}}{3}+\frac{S_{(4)}}{4}-\frac{S_{(5)}}{5}+\frac{S_{(6)}}{6}-\frac{\zeta_{(7)}}{7}+\frac{S_{(8)}}{8}=0.6284 \ldots \quad \therefore \mathrm{R}=1-\ln 2+\mathrm{r}<0.6284 \cdots$.

$\mathrm{r}<0.6284 \cdots+\ln 2-1=0.321520 \cdots<1 / 3$. Note [7]: $\ln 2=1-1 / 2+1 / 3-1 / 4+1 / 5-1 / 6+\cdots+\left[(-1)^{\mathrm{n}-1}\right] / \mathrm{n}$. Euler's constant $r$ since have no one to prove whether it is rational, now to prove - Euler number frequency constant $r$ is irrational.

In (3) type $R=1-\ln 2+r<1 / 3$, Obviously,this shows that the Euler constant $R$ and $r$ are two different irrational numbers .Finished.

\section{The Riemann zeta Function Principle: $\mathbf{s}$ for Arbitrary Number; $\mathbf{S}-\mathbf{u p}, \mathbf{p}$ for Prime Numbers}

$$
\zeta(\mathrm{sn})=1+1 / 2 \mathrm{~s}+1 / 3 \mathrm{~s}+1 / 4 \mathrm{~s}+\ldots . . .+1 / \mathrm{ns}>1>\Pi(1-\mathrm{p}-\mathrm{s})^{-1} .
$$

Prove: (1).let $\zeta(\mathrm{s} 1)=1+1 / 2 \mathrm{~s},(1 / 2 \mathrm{~s}) \zeta(\mathrm{s} 1)=1 / 2 \mathrm{~s}+1 / 4 \mathrm{~s}$,

[8] namely $\left(1-1 / 2^{s}\right) \zeta\left(s_{1}\right)=\zeta\left(s_{1}\right)-1 / 2^{s} \zeta \quad\left(s_{1}\right)=1-1 / 4 s^{s}$; (2).let $\zeta \quad\left(s_{2}\right)=1+1 / 2^{s}+1 / 3^{s}$, so

$\zeta\left(\mathrm{s}_{2}\right) / 2^{\mathrm{s}}=1 / 2^{\mathrm{s}}+1 / 4^{\mathrm{s}}+1 / 6^{\mathrm{s}}, \zeta \quad\left(\mathrm{s}_{2}\right) /\left(1-1 / 2^{\mathrm{s}}\right)=1 / 2^{\mathrm{s}}+1 / 4^{\mathrm{s}}+1 / 6^{\mathrm{s}}, 1 / 3^{\mathrm{s}}\left(1-1 / 2^{\mathrm{s}}\right) \zeta \quad\left(\mathrm{s}_{2}\right)=1 / 3^{\mathrm{s}}+1 / 9^{\mathrm{s}}-1 / 12^{\mathrm{s}}-1 / 18^{\mathrm{s}}$, $\left(1-1 / 3^{s}\right)\left(1-1 / 2^{s}\right) \zeta \quad\left(s_{2}\right)=1-1 / 4^{s}-1 / 6^{s-1}-9^{s}+1 / 12^{s}+1 / 18^{s}+1 / 24^{s}$. (3). let $\zeta\left(s_{3}\right)=1+1 / 2^{s} s+1 / 3^{s} s+1 / 4 s^{s} s ;$

$\zeta\left(s_{3}\right) / 2^{s}=1 / 2^{s} s+1 / 4^{s}+1 / 6^{s} s+1 / 8^{s}, 1 / 3^{s} \zeta\left(s_{3}\right)=1 / 3^{s}+1 / 9^{s}-1 / 18^{s}-1 / 24 s,\left(1-1 / 3^{s}\right)\left(1-1 / 2^{s}\right)$

$\left(\mathrm{s}_{3}\right)=1-1 / 6^{\mathrm{s}}-1 / 8^{\mathrm{s}}-1 / 9^{\mathrm{s}}+1 / 18^{\mathrm{s}}+1 / 24^{\mathrm{s}}$.

$\left(1-1 / 4^{\mathrm{S}}\right)\left(1-1 / 3^{\mathrm{S}}\right)\left(1-1 / 2^{\mathrm{S}}\right) \quad \zeta\left(\mathrm{s}_{3}\right)=1-1 / 4^{\mathrm{S}}-1 / 6^{\mathrm{S}}-1 / 8^{\mathrm{S}}-1 / 9^{\mathrm{S}}+1 / 18^{\mathrm{S}}\left(1-1 / 4^{\mathrm{S}}\right)+1 / 24^{\mathrm{S}}\left(1-1 / 4^{\mathrm{S}}\right)$;

(4).

(5). let $\zeta\left(s_{n}\right)=1+1 / 2^{s}+1 / 3^{s}+1 / 4^{s} \ldots . . .+1 / n^{s}, \ldots \ldots \ldots . .$,

In turn have $1 / 2^{\mathrm{s}} \zeta\left(\mathrm{s}_{\mathrm{n}}\right)=1 / 2^{\mathrm{s}}+1 / 4^{\mathrm{s}}+1 / 6^{\mathrm{s}}+1 / 8^{\mathrm{s}}+\ldots+1 /(2 \mathrm{n})^{\mathrm{s}}, \ldots \ldots .$.

Above 5 step derivation shows that Euler first conclusion namely elimination all even term is not set up; The second conclusion elimination all multiples of 2 and 3; The third repeat of the last conclusion is equal to 1 . Because the one on the right: increase the negative even term and are not even equal to and less than 1; The last important: if the increase of negative even term is even with the increase of the sum and approximate as 0 , so the original type zeta don't (s), available

$S=$ up, $1+1 / 2^{s+} / 3^{s}+1 / n^{s+.}=1$. The paradox arises when $s=1$, therefore Euler's turn to the right of the conclusion as 1 to deal with, only his personal subjective not objective facts. Summarize the above argument, to prove that the number and frequency that the laws of Riemann zeta function. The end.

\section{The Riemann Hypothesis Is not Equality}

The relations between the classical Euler's constant and Riemann's function are as follows:[9] 


$$
\mathrm{R}=\sum_{\mathrm{n}=2}^{\infty} \frac{(-1)^{n} \varsigma_{(n)}}{n}=\ln \left(\frac{4}{\pi}\right)+\sum_{n=1}^{\infty} \frac{(-1)^{\mathrm{n}-1} \varsigma_{(\mathrm{n}+1)}}{2^{n}(n+1)}
$$

Very honored! The expression of $\mathrm{R}$ has been replaced by the Euler number frequency constant, if not in the same relationship and other data, then only the Riemann function is no longer the original nature of conjecture - Riemann conjecture is not normal.It leads to the new discovery of incomplete Riemann's function. Professor successfully solved the problem of Riemann's conjecture Mathematics Education People's network [10]: In November 19, 2015, according to the British "Daily Mail" reported in November 17th, recently, Professor Enoch Opie Yemi, Professor of Nigeria in Nigeria, a university. He said he made a key breakthrough in 2010, which will be able to solve the problem for the one thousand years to lay the foundation. He said..., it is because the students believe in themselves, he began to try to solve this math problem. However, clay Mathematics Institute has neither confirmed nor denied Dr. Enoch formally solved this problem, just a simple representation of the Millennium mathematics problem solution not comment. In this (9) type of point of view the above reports distortion.

\section{New Discovery of Incomplete Riemann's Function}

The law of the number and frequency of science 4 :

The general term Un is monotone decreasing, infinite monotonically increasing series and Sn or convergence or divergence, $\mathrm{Sn}$ all odd terms and $\mathrm{S}$ odd number greater than all even terms and $\mathrm{S}$ even number. Record for $\mathrm{S}_{\text {odd }}>\mathrm{Sn} / 2>\mathrm{S}_{\text {even. }}$

The number of frequency to prove scientific law 4: Sn series because of increasing, known general Un monotonically decreasing, is set to $\mathrm{Un}>\mathrm{Un}+1$, the total $\mathrm{U} 1>\mathrm{U} 2>\mathrm{U} 3>\ldots>\mathrm{Un}-1>\mathrm{Un} \mathrm{Sn}=\mathrm{S}_{\text {odd }}+\mathrm{S}_{\text {even..., , }}$

$\mathrm{S}_{\text {odd }}=\mathrm{U} 1+\mathrm{U} 3+\mathrm{U} 5+\ldots>\mathrm{S}_{\text {even }}=\mathrm{U} 2+\mathrm{U} 4+\mathrm{U} 6+\ldots . ., \mathrm{Sn} / 2=[\mathrm{U} 1+\mathrm{U} 3+\mathrm{U} 5 \ldots+\mathrm{Un}] / 2+[(\mathrm{U} 2++\mathrm{U} 4+\mathrm{U} 6)+\ldots+\mathrm{U}(\mathrm{n}-1)] / 2$

$>[\ldots(\mathrm{U} 2++\mathrm{U} 4+\mathrm{U} 6+\ldots+\mathrm{U}(\mathrm{n}-1)] / 2+[(\mathrm{U} 2++\mathrm{U} 4+\mathrm{U} 6+\ldots+\mathrm{U}] / 2=2[(\mathrm{n}-1)(+\mathrm{U}(\mathrm{n}-1)] / 2 \mathrm{U} 2++\mathrm{U} 4+\mathrm{U} 6+\ldots=\mathrm{S} 2$.

$\mathrm{S}_{\text {odd }}>\mathrm{Sn} / 2>\mathrm{S}_{\text {even. }} \quad$ Finished.

Example: $1=1 / 2+1 / 2=1 / 2+1 / 4+1 / 8+\ldots=S n . ., \quad S_{\text {odd }}=1 / 2+1 / 8+1 / 32+\ldots=2 / 3, S_{\text {even }}=1 / 4+1 / 16+1 / 64+\ldots$ $=1 / 3$.

$\mathrm{S}$ odd $+\mathrm{S}$ even $=\mathrm{Sn}, \mathrm{S}_{\text {odd }}>\mathrm{Sn} / 2>\mathrm{S}$ even.

$$
\varsigma_{(S)}=\prod_{P, \text { Prime }} \frac{1}{1-p^{-s}} \text {. when } \mathrm{s}=2 \text {,so } \varsigma_{(2)}=1+\frac{1}{2^{2}}+\frac{1}{3^{2}}+\frac{1}{4^{2}}+\ldots+\frac{1}{\mathrm{n}^{2}}=\sum_{n=1}^{\infty} \frac{1}{n^{2}}=\frac{\pi^{2}}{6}
$$

By using the Fourier series expansion of the function, sometimes it can get some special and, for example, by the results of 5 examples, there are

$$
f(x)=|x|=\frac{\pi}{2}-\frac{4}{\pi} \sum_{k=1}^{\infty} \frac{1}{(2 k-1)^{2}} \cos (2 k-1) x,(-\pi \leq x \leq \pi)
$$

let $\mathrm{x}=0, \sum_{k=1}^{\infty} \frac{1}{(2 k-1)^{2}}=\frac{\pi^{2}}{8}$.

The zeta (2) when the number of frequency (assuming)

$$
1+\frac{1}{2^{2}}+\frac{1}{3^{2}}+\frac{1}{4^{2}}+\ldots+\frac{1}{\mathrm{n}^{2}}=\sum_{n=1}^{\infty} \frac{1}{n^{2}}=\frac{\pi^{2}}{6}=\varsigma_{(2)}=\sigma
$$


The following two expressions are incomplete

$$
\sigma_{2}=\frac{1}{2^{2}}+\frac{1}{4^{2}}+\frac{1}{6^{2}}+\ldots=\frac{\sigma}{4}=\frac{\sigma_{1}+\sigma_{2}}{4}=\frac{\pi^{2}}{24}, \sigma_{1}=1+\frac{1}{3^{2}}+\frac{1}{5^{2}}+\frac{1}{7^{2}}+\ldots=\frac{\pi^{2}}{8}
$$

Let $\mathrm{n}=2 \mathrm{~m}, \mathrm{~m} \rightarrow \infty$.

Solving:

$$
\sigma_{2}=\frac{1}{2^{2}}+\frac{1}{4^{2}}+\frac{1}{6^{2}}+\ldots+\frac{1}{(2 \mathrm{~m})^{2}}=\frac{\pi^{2}}{24}-R_{2}
$$

$$
\begin{aligned}
& \frac{\sigma}{4}=\frac{1}{4}\left(1+\frac{1}{2^{2}}+\frac{1}{3^{2}}+\ldots+\frac{1}{(2 \mathrm{~m})^{2}}\right) \\
\because & =\frac{1}{2^{2}}+\frac{1}{2^{4}}+\frac{1}{6^{2}}+\ldots+\frac{1}{(2 m)^{2}}+\frac{1}{(2 m+1)^{2}}+\ldots+\frac{1}{4(2 m)^{2}} \\
& =\sigma_{2}+R_{2},
\end{aligned}
$$

Total 2 items, among

$$
\begin{gathered}
R_{2}=\frac{1}{(2 m+1)^{2}}+\ldots+\frac{1}{4(2 m)^{2}}, \\
\therefore \sigma_{2}=\frac{1}{2^{2}}+\frac{1}{4^{2}}+\frac{1}{6^{2}}+\ldots+\frac{1}{4 m^{2}}=\frac{\sigma}{4}-R_{2}=\frac{\pi^{2}}{24}-R_{2}
\end{gathered}
$$

Total M Even term,

$$
\because 1-\frac{1}{2}+\frac{1}{3}-\frac{1}{4}+\frac{1}{5}-\ldots+(-1)^{2 \mathrm{n}-1} \frac{1}{2 n}=\frac{1}{\mathrm{n}+1}+\frac{1}{n+2}+\ldots+\frac{1}{2 n}=\ln 2 \neq 0,
$$

On both sides of the square, too

$$
\begin{aligned}
{[1-} & \left.\frac{1}{2}+\frac{1}{3}-\frac{1}{4}+\frac{1}{5}-\ldots+(-1)^{2 \mathrm{n}-1} \frac{1}{2 n}\right]^{2}=\left[\frac{1}{\mathrm{n}+1}+\frac{1}{n+2}+\ldots+\frac{1}{2 n}\right]^{2}=(\ln 2)^{2}, \mathrm{n} \rightarrow \infty \\
& (\ln 2)^{2}=\left[\frac{1}{\mathrm{n}+1}+\frac{1}{n+2}+\ldots+\frac{1}{2 n}\right]^{2}=\left(\frac{1}{\mathrm{n}+1}\right)^{2}+\left(\frac{1}{n+2}\right)^{2}+\ldots+\left(\frac{1}{2 n}\right)^{2}+R_{2}^{\prime \prime} \\
\therefore & >4\left[\frac{1}{(2 n+1)^{2}}+\frac{1}{(2 n+2)^{2}}+\ldots+\frac{1}{4(2 n)^{2}}\right]+R_{2}^{\prime \prime} \\
> & 4 R_{2}+R_{2}^{\prime \prime},
\end{aligned}
$$




$$
\begin{gathered}
(\ln 2)^{2}>4 R_{2}+R_{2}^{\prime \prime}, R_{2}^{\prime}<\frac{(\ln 2)^{2}}{4}<\frac{1}{8} . \\
\because R_{2}^{\prime \prime} \rightarrow \text { fast } \rightarrow 0, \frac{1}{\mathrm{n}^{2}} \rightarrow 0, \therefore R_{2} \rightarrow \frac{(\ln 2)^{2}}{4} . \\
\sigma_{1}=\sigma-\sigma_{2}=1+\frac{1}{2^{2}}+\frac{1}{3^{2}}+\frac{1}{4^{2}}+\ldots+\frac{1}{(2 \mathrm{~m})^{2}}-\sigma_{2}=\frac{\pi^{2}}{6}-\frac{\pi^{2}}{24}-\mathrm{R}_{2}=\frac{\pi^{2}}{8}-\mathrm{R}_{2}, \\
\sigma_{3}=\sigma_{1}-\sigma_{2}=\frac{\pi^{2}}{8}+R_{2}-\frac{\pi^{2}}{24}-R_{2}=1-\frac{1}{2^{2}}+\frac{1}{3^{2}}-\frac{1}{4^{2}}+\ldots=\frac{\pi^{2}}{12} . \text { end. }
\end{gathered}
$$

\section{Discussion}

Zeta (2) the number of frequency constant discovery is inevitable. According to the frequency of the fourth law, can determine the corresponding constant P series one by one, call function is a constant frequency P series. No details.

\section{Reference}

[1] Websnapshot.

Retrieved

from

the

website: http://snapshot.sogoucdn.com/websnapshot?ie=utf8\&url=http\%3A\%2F\%2Fbaike.baidu

[2] (2015). A number of smaller than a given value of the primes (Riemann proposed the Riemann conjecture - Xie Guofang's original paper). The Number of Language of Light.

[3] Euler_product_formula. Retrieved from the website: http://www.changhai.org/articles/science/mathematics/euler_product_formula.php

[4] Sogoucdn. Retrieved from the website: http://snapshot.sogoucdn.com/websnapshot?ie=utf8\&url=http\%3A\%2F\%2Fbaike.sogou

[5] Wu, H. F. (2015). The Number and Change Rule of Science. Changchun: Ji Lin University Press.

[6] Song, W. Z. (2015). The recurrence formula of the sum of p-series ( $p$ is even). Studies College Mathemmatics[J], 18(3), 10-11.

[7] nongyegongye. $\quad$ Retrieved from http://www.book118.com/nongyegongye/html/81540.html

[8] Wu, H. F. (2015). The Number and Change Rule of Science. Changchun: Ji Lin University Press.

[9] Lu, C. H. (2012). The Riemann Hypothesis Ramble. Tsinghua University Press.

[10] (2015). Nigeria Professor Successfully Solved the World Famous Puzzle Riemann Conjecture. NetEase News.

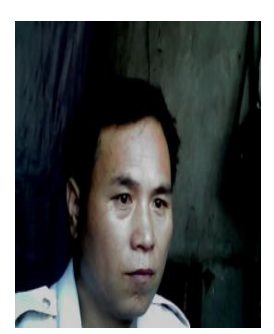

Wu Hefa was born on October 2, 1972. He is the number and change rule of science of founder, Shandong heze zhongxing town wu ji village, zip code 274329. His research direction is frequency of science. 